\title{
AUTOMORPHISMS OF TORSION-FREE NILPOTENT GROUPS OF CLASS TWO
}

\author{
MANFRED DUGAS AND RÜDIGER GÖBEL
}

\begin{abstract}
We construct 2-divisible, torsion-free abelian groups $G$ admitting an alternating bilinear map. We use these groups $G$ to find nilpotent groups $N$ of class 2 such that $\operatorname{Aut}(N)$ modulo a natural normal subgroup is a prescribed group.
\end{abstract}

\section{INTRODUCTION}

The main problem in working with torsion-free nilpotent groups seems to be the lack of elementary methods in constructing these groups. Wreath products are only useful for finite $p$-groups, cf. [5] and (upper triangular) matrix methods become too complicated for dimensions greater than 3. Hence many authors restrict their interest to dimension 3 and construct nilpotent groups of class 2 , see $[6,11,12,16,18]$ for example. We will do the same and discuss their results in connection with our new construction below. The matrix methods have another defect as well. The outcoming nilpotent groups are strongly related with the underlying ring as well as the shape of the upper triangular 3-by-3 matrices, i.e. with the matrix multiplication. Hence strong ring theoretic results on $E$-rings are needed to get the desired nilpotent groups [6].

Here we will use a different technique due to Baer [1] and published over sixty years ago in the Transactions of the AMS. A good account on this can be found in Warfield [22, pp. 26-42], and we recall some basic facts. Consider the central extension $G$ of the abelian groups $A, B$,

$$
1 \longrightarrow A \stackrel{\text { id }}{\longrightarrow} G \longrightarrow B \longrightarrow 1
$$

(where $A \subseteq z G$, the center of $G$ ). If $x^{\prime}, y^{\prime} \in G$ are preimages of $x, y \in B$, then the commutator $\left[x^{\prime}, y\right]$ is in $A$ and independent of the choice of the preimages of $x, y$. Using Hall's commutator formulas it is easy to see that the commutator map

$$
f:[,]: B \times B \rightarrow A
$$

is an alternating bilinear map, which codes the multiplication on $G$.

Conversely, any such alternating bilinear map arises from some central extension $(*)$, which is Baer's theorem [1], extended by Lazard [14], cf. [14] and Warfield [22, p. 41, Theorem 5.14].

Received by the editors November 2, 1989 and, in revised form, May 10, 1990.

1980 Mathematics Subject Classification (1985 Revision). Primary 20F18, 20K20. 
Theorem 1.0. Let $G^{+}$be an abelian group which is uniquely 2-divisible, equipped with an alternating bilinear map $f: G \times G \rightarrow G$ such that $f(f(x, y), z)=0$ for all $x, y, z \in G$.

Define a multiplication on $G$, letting

$$
x y=x+y+\frac{1}{2} f(x, y) .
$$

then $G^{\cdot}=G_{f}^{\cdot}$ is a nilpotent group of class 2 which is uniquely 2-radicable and $\left(G^{+}, f\right) \rightarrow G_{f}^{\cdot}$ is a category isomorphism.

In particular, automorphisms of $G_{f}^{\cdot}$ correspond to automorphisms of $\left(G^{+}, f\right)$, which is crucial for our investigation. We have the following consequence of the Baer-Lazard Theorem 1.0 (with a somewhat weaker hypothesis).

Proposition 1.1. Let $G^{+}$be a 2-torsion-free abelian group, equipped with an alternating bilinear map

$$
f^{\prime}: G \times G \rightarrow G \text { such that } f(f(x, y), z)=0 \quad \text { for all } x, y, z \in G .
$$

Define the nilpotent group $G_{f}^{*}$ of class 2 by letting $x . y=x+y+f(x, y)$. Then the following are equivalent.

(1) $\alpha \in$ Aut $G_{f}^{*}$,

(2) $\alpha \in$ Aut $G^{+}$and $\alpha$ is $f$-invariant, i.e., $f(x, y) \alpha=f(x \alpha, y \alpha)$ for all $x$, $y \in G$.

We will give an easy and direct proof of this result, which is the starting point of our investigations. Recall that $G^{+}$is 2-torsion-free, if the 2-socle $G[2]=\{g \in G: 2 g=0\}$ is 0 .

Proof. (1) $\rightarrow(2)$. Using the multiplication of $G_{f}^{\cdot}$, the commutator of $x, y \in$ $G$ equals $[x, y]=2 f(x, y)$, hence

$$
2 f(x, y) \alpha=(2 f(x, y)) \alpha=[x, y] \alpha=[x \alpha, y \alpha]=2 f(x \alpha, y \alpha) .
$$

We derive

$$
f(x, y) \alpha=f(x \alpha, y \alpha)
$$

from $G[2]=0$ and $\alpha$ is $f$-invariant.

Using this and $f(x, y) \in z G$; also

$$
\begin{aligned}
(x+y) \alpha & =(x \cdot y-f(x, y)) \alpha=(x \cdot y \cdot(-f(x, y))) \alpha \\
& =(x \alpha) \cdot(y \alpha) \cdot-f(x, y) \alpha=(x \alpha) \cdot(y \alpha)-f(x, y) \alpha \\
& =x \alpha+y \alpha+f(x \alpha, y \alpha)-f(x, y) \alpha=x \alpha+y \alpha
\end{aligned}
$$

and $\alpha \in$ Aut $G^{+}$.

$(2) \rightarrow(1)$ is trivial, and Proposition 1.1 is shown.

Let $\left(G^{+}, f\right)$ be as in Proposition 1.1. Then we will say that $f$ is not degenerate, if for any $x \in G \backslash f(G, G)$ there exists $y \in G^{*}$ such that $f(x, y) \neq 0$.

If $G=G_{f}^{*}$ is as in Proposition 1.1 and if $g \in G^{*}$, then clearly $f_{g}=f(, g)$ is an endomorphism of $G^{*}$ and we may consider $f_{X}=\left\{f_{X}: x \in X\right\}$ for any subset $X$ of $G$. The same argument as in (1.1) then shows 
Lemma 1.2. Let $(G, f)$ and $G_{f}^{*}$ be as in Proposition 1.1. Then the following hold.

(a) $f(G \times G) \subseteq z G_{f}^{\cdot}$.

(b) If $f$ is not degenerate, then $z G_{f}^{\cdot}=f(G, G)$, and under the same assumption follows

Lemma 1.3. Inn $G^{*}=1+f_{2 G}$.

The aim of this paper is a realization theorem for prescribed outer automorphism groups of nilpotent groups of class 2. In view of Proposition 1.1 our method should be clear. We will model abelian groups $G^{+}$with an alternating bilinear map $f$ such that the $f$-invariant automorphisms of $G^{+}$do "almost" what we want.

After these reduction arguments we will describe our abelian-bilinear methods which hopefully will motivate nonabelian algebraists to study recent techniques from abelian group theory which seem to be useful in many areas of algebra.

Let $H$ be the group which we want to realize as an outer automorphism group. Taking a cotorsion-free, commutative ring $S$ (any reduced subring of $\mathbb{Q}$ will suffice), we convert $H$ into a group ring $R=S[H]$ which is cotorsion-free as well; see $\S 2$ for cotorsion-freeness. Next we define an alternating bilinear map $f$ on a free $R$-module $F$ (where $F$ is large enough to allow some combinatorial arguments). The map $f$ is then defined naturally on a basis of $F$. The module $F$ can be viewed as a dense submodule of its $\mathbb{Z}$-adic completion $F$ and an easy continuity argument shows that $f$ can be extended to an alternating bilinear map $\hat{f}$ of $\widehat{F}$, which we denote by $f$ as well.

In $\S 2$ we derive some useful properties of $f$. The main result in $\S 4$ on $R$ modules is an application of Shelah's Black Box [21] to $(\widehat{F}, f)$; see [3] for a proof of the Black Box. This will yield an $R$-module $G \subseteq \widehat{F}$ containing $F$ such that

$$
\text { End } G=R \oplus f_{G},
$$

where $f_{G}$ is an ideal of End $G$ and $(* *)$ is a split extension. The category isomorphism, given in Proposition 1.0, applied to $\left(G^{+}, f\right)$ with $(* *)$ leads to the desired torsion-free nilpotent group $G_{f}^{*}=G^{*}$ of class 2 . This functor has the following consequences.

Theorem 3.1. Given a group $H$, then there exists a torsion-free nilpotent group $G^{*}$ of class two such that the following holds.

$$
\text { Aut } G^{*}=\left(1 \times f_{G}\right) \rtimes H^{*} \text { is as semidirect product }
$$

and $1+f_{G}=\operatorname{Stab} G^{*}$ is the stabilizer of the central series with

Stab $G^{*}=\left\{\sigma \in\right.$ Aut $G^{*}: \sigma \mid z G^{*}=1$ and the induced $\left.\sigma \mid G^{*} / z G^{*}=\mathrm{id}\right\}$.

The group $H^{*}$ is a subgroup of the units $U R$ of $R=S[H]$ consisting of all $r \in U R$ with $r^{\#}=1$ and $r^{*}=1$. If $r=\sum_{g \in H} g r_{g}\left(r_{g} \in S\right)$, then $r^{\#}=\sum_{g \in H} r_{g}$ defines the natural ring epimorphism $R \rightarrow S$ and $r^{*}=\sum_{g \in H}\left(g^{-1}\right) r_{g}$ defines an antiautomorphism of $R$.

In [5 and 7] we obtained similar results for locally soluble groups with " $H^{*}$ " resp. $1+f_{G}$ replaced by $H$, resp. Inn $G^{\circ}$. It follows from (1.3) or from 
$1+f_{G}=\operatorname{Stab} G^{*}$ that $\operatorname{Inn} G^{*}$ is a subgroup of $1+f_{G}$, and clearly $H \subseteq H^{*}$ from $h^{\#}=1=h \cdot h^{*}$ for all $h \in H \subseteq U R$. Hence it is desirable to have each pair Inn $G^{*} \subseteq 1+f_{G}$ and $H \subseteq H^{*}$ essentially equal.

A general theorem of Hall's [9] implies that Stab $G^{*}$ is abelian and we are able to show

Corollary 3.2. If $S=\mathbb{Z}$, then $H=H^{*}$ and Aut $G^{*}=\operatorname{Stab} G^{*} \rtimes H$, where Stab $G^{*} /$ Inn $G^{*} \cong \bigoplus Z_{2}$ is an elementary abelian 2-group of rank $|G|$.

Hence we can prescribe outer automorphisms Out $G=$ Aut $G^{*} / \operatorname{Inn} G$ up to $\bigoplus Z_{2}$. Moreover,

Corollary 3.3. If $S=\mathbb{Z}_{(2)}$ (localized at $2 \mathbb{Z}$ ), then Aut $G^{*}=\operatorname{Inn} G^{*} \rtimes H^{*}$ for all $H^{*}$, which is a (true) realization theorem for $H^{*}$.

If $H$ is not too complicated, then we can determine $H^{*}$ in case (3.3), see $\S 5$. We have $V_{4}^{*} \cong V_{4} \oplus Z_{2}, Z_{3}^{*}=Z_{3}(\S 5)$ and $1^{*}=1$. From $1^{*}=1$ we obtain a class (not a set) of nilpotent groups of class 2 which are semicomplete (Out $G=1$ ).

The papers $[5,6,7,11,12,18,19,23,24]$ in the references deal with the same topic and we briefly discuss their relationship. In $[5,7]$ we consider mainly locally finite $p$-groups and arbitrary groups, respectively. These classes being closed under sufficiently many operators, allow a class of groups $G$ with Aut $G=\operatorname{Inn} G \rtimes H$. If $H=1$, then $G$ provides an answer to a question of Philip Hall's. The constructions are purely based on group theory; we mainly use topological wreath products, which are very different from the methods in this paper. [6] uses matrix methods as indicated at the beginning. Method and results in [6] extend [24]. In [6] we are unable to determine Out $G$ as in (3.2) or (3.3), however we also derive a similar result to (3.1) by using the $E$-ring approach. Heineken [11] constructed nilpotent groups $G$ of class 2 by using Frucht's realization of groups as automorphism groups of graphs. He codes a given graph $\Gamma$ into $G$ by using divisibility by primes. Hence all automorphisms of $G$ act on $\Gamma$ and this leads to realization theorems of Aut $G / \operatorname{Aut}_{c} G$, where $\mathrm{Aut}_{c} G$ is the subgroup of all central automorphisms $\{\sigma \in$ Aut $G$ with $g \sigma^{*}=g^{-1}\left(g^{\sigma}\right)=1$ for all $\left.g \in G\right\}$. However, observe that Stab $G \subseteq \operatorname{Aut}_{c} G$ and we have not equality in general. In this sense (3.2) and in particular (3.3) are stronger results. These methods have been extended in [12] and more recently in [18, 19, 23]. They are used in [19] to sharpen Schupp's result [20], which characterizes inner automorphisms as those automorphisms which can be extended to any group extension. This is essentially the case $H=1$ in [7] or (3.2) for class 2. This result also holds within the class of finite groups [18], and a more elementary proof is given in [19].

We would like to thank the referee for very useful comments and for drawing our attention to the papers [13, 17] dealing with a similar question. In [13] all finite groups are realized as outer automorphism groups of some discrete subgroups of $\operatorname{PSL}(2, C)$. Using the Bass-Serre theory of group actions on trees and graphs, the result was extended in [17] to arbitrary groups, which was the main result in [7].

\section{ABELIAN GROUPS WITH ALTERNATING BILINEAR MAP}

Let $H$ be a group and $S$ a cotorsion-free ring. Recall that $S$ is cotorsion-free if the additive group $S^{+}$is cotorsion-free, cf. [4]. Moreover $S^{+}$is cotorsion- 
free if and only if $\operatorname{Hom}(\widehat{\mathbb{Z}}, S)=0$, with $\mathbb{Z}=\prod_{p} J_{p}$ the $\mathbb{Z}$-adic completion of $\mathbb{Z}$. This is the case if and only if $S^{+}$is torsion-free, reduced ( $\mathbb{Q}$ is not a subgroup of $S^{+}$) and $J_{p}$ is not a subgroup of $S^{+}$for any prime $p ; J_{p}$ denotes the $p$-adic integers.

Next we will consider the group ring $R=S[H]$. It is easy to see that $R$ is cotorsion-free as well. If $r=\sum_{g \in H} g z_{g}$ with $z_{g} \in S$, let

(i) ${ }^{\#}: R \rightarrow S$ be the augumentation map $r^{\#}=\sum_{g \in H} z_{g}$, which is a ring epimorphism, and let

(ii) $^{*}: R \rightarrow R$ be given by $r^{*}=\sum_{g \in H}\left(g^{-1}\right) z_{g}$, which is an antiautomorphism of the ring $R$.

If $\lambda$ is an (infinite) cardinal, we will use the tree $T={ }^{\omega>} \lambda$ of all maps $\pi: n \rightarrow \lambda$ for all $n \in \omega$. The tree has a natural partial order, defined by inclusion of maps.

$$
\tau \subseteq \sigma \Leftrightarrow \operatorname{dom} \tau \leq \operatorname{dom} \sigma \quad \text { and } \quad \sigma \mid \operatorname{dom} \tau=\tau .
$$

Moreover, we will use the lexicographical (linear) order on $T$, which we denote by " $\leq$ or $<$."

We define two free $S$-modules on the tree $T$, taking

$$
\begin{gathered}
F_{1}=\bigoplus_{\tau g} e_{\tau g} S \text { freely generated by } e_{\tau g}(\tau g \in T \times H) \text { and } \\
F_{0}=\bigoplus_{\substack{\tau \leq \sigma \in T \\
g \in H}} d_{\tau \sigma g} S \text { freely generated by } d_{\tau \sigma g}(\tau \sigma g \in T \times T \times H, \tau \leq \sigma) .
\end{gathered}
$$

Using (i), we make $F_{0}, F_{1}$ into $R$-modules. Let $r=\sum_{g \in H} g z_{g} \in R$ as above, and define

$$
e_{\tau x} r=\sum_{g \in H} e_{\tau x g} z_{g} \quad \text { for any } \tau x \in T \times H
$$

and

$$
d_{\tau \sigma x} r=d_{\tau \sigma x}\left(r^{\#}\right) \quad \text { for any } \tau \sigma x \in T \times T \times H \text { with } \tau \leq \sigma .
$$

Note that $d_{\tau \sigma x} y=0$ for all $y \in \operatorname{ker}^{\#}$. Next we define an alternating bilinear map $f$ on $F=F_{0} \oplus F_{1}$ :

$$
f: F \times F \rightarrow F \text { with } f(f(x, y), z)=0 \quad \text { for all } x, y, z \in F .
$$

Define $f: F_{1} \times F_{1} \rightarrow F_{0}$ by setting

$$
f\left(e_{\alpha g}, e_{\beta h}\right)= \begin{cases}0 & \text { if } \alpha=\beta, \\ d_{\alpha \beta h^{-1}} & \text { if } \alpha<\beta, \\ -d_{\beta \alpha h g^{-1}} & \text { if } \alpha>\beta,\end{cases}
$$

for all $\alpha, \beta \in T, g, h \in H$. If we also let $f \mid F_{0} \times F=0$, then $f$ is well defined on an $S$-basis of $F$ and this map extends uniquely to an alternating $S$ bilinear map $f$ on $F$. Moreover $f(F \times F) \subseteq F_{0} \subseteq F$. We will not distinguish between $f$ and $f \mid F_{1} \times F_{1}: F_{1} \times F_{1} \rightarrow F_{0}$ in the following. This bilinear map and the $R$-module $F$ will be fixed throughout this paper. The additional condition $f(f(x, y), z)=0$ follows immediately from $f(F \times F) \subseteq F_{0}$ and $f\left(F_{0} \times F\right)=0$.

We want to derive some elementary properties of $f$. 
Lemma 2.1. For $x, y \in F_{1}$ and $r \in R$, we have $f(x r, y)=f\left(x, y r^{*}\right)$. Proof. We may restrict ourselves to generating elements of $F$. Let

$$
x=e_{\alpha g}, \quad y=e_{\beta h} \quad \text { with } \alpha<\beta \text { and } r \in H .
$$

Then, using (ii) and $(*)$, we have

$$
\begin{aligned}
f\left(e_{\alpha g} r, e_{\beta h}\right) & =f\left(e_{\alpha g r}, e_{\beta h}\right)=d_{\alpha \beta g r h^{-1}}=d_{\alpha \beta g\left(h r^{-1}\right)^{-1}}=f\left(e_{\alpha g}, e_{\beta h r^{-1}}\right) \\
& =f\left(e_{\alpha g}, e_{\beta h} r^{-1}\right)=f\left(e_{\alpha g}, e_{\beta h} r^{*}\right) .
\end{aligned}
$$

Lemma 2.1 can be extended by a continuity argument.

Lemma 2.2. There exists a unique extension $\hat{f}: \widehat{F} \times \widehat{F} \rightarrow \widehat{F}$ of $f$, satisfying the following conditions:

(i) $\hat{f}$ is an alternating $S$-bilinear map on $\widehat{F}$.

(ii) $\hat{f}\left\lceil\widehat{F}_{0} \times \widehat{F}=0\right.$.

(iii) $\hat{f}(x r, y)=\hat{f}\left(x, y r^{*}\right)$ for all $x, y \in \widehat{F}, r \in R$.

Proof. At the beginning of this section we observed that $R=S[H]$ is cotorsionfree, which implies that $F$ is cotorsion-free. The $\mathbb{Z}$-adic topology of $F$ must be Hausdorff and $f: F_{1} \times F_{1} \rightarrow F_{0}$ is a continuous map by linearity, which extends uniquely to a bilinear map $\hat{f}: \widehat{F}_{1} \times \widehat{F}_{1} \rightarrow \widehat{F}_{0}$. We must show that $\hat{f}$ satisfies (i), (ii) and (iii). However, $f$ is an alternating bilinear map on the dense subset $F$ with $f \mid F_{0} \times F=0$, hence (i) and (ii) follow by continuity. Similarly, (iii) follows from Lemma 2.1 .

Remark. Again, we will identify $\hat{f}$ and $f$ with $\hat{f}\left\lceil\widehat{F}_{1} \times \widehat{F}_{1} \rightarrow \widehat{F}_{0}\right.$.

Next we determine particular values of $f$.

Lemma 2.3. If $x=e_{\alpha h} \pi \in \widehat{F}_{1}$, with $\pi \in\langle\hat{1}\rangle \subseteq \widehat{R}$, then $f(x, x r)=0$ for all $r \in R$.

Proof. Suppose $r=\sum_{g} g z_{g} \in R=S[H]$. Using (*) for $\alpha=\beta$ and continuity, we have

$$
f(x, x r)=f\left(e_{\alpha h} \pi, \sum_{g} e_{\alpha h g} \pi z_{g}\right)=\sum_{g} f\left(e_{\alpha h}, e_{\alpha h g}\right) \pi^{2} z_{g}=0 .
$$

If $x=\sum_{\alpha g} e_{\alpha g} x_{\alpha g} \in \widehat{F}_{1}$, we define a (unique) support

$$
[x]=\left\{\alpha \in T: x_{\alpha g} \neq 0 \text { for some } g \in H\right\} \text {, }
$$

which is a countable subset of $T$. Similarly we define a support of elements in $\widehat{F}_{0}$. Then we have the following lemma.

Lemma 2.4. Suppose $x \in \widehat{F}_{1}$ and $a=\sum_{n \in \omega} e_{\alpha_{n} 1} a_{n}$ with $a_{n} \neq 0$ for all $n \in \omega$ such that $[a] \cap[x]$ is finite. Then we have

$$
f(a, x)=0 \Leftrightarrow x=0 \text {. }
$$

Proof. If $x=0$, clearly $f(a, x)=0$. Conversely, if $x=\sum_{\beta g} e_{\beta g} x_{\beta g}$, then

$$
\begin{aligned}
f(a, x) & =f\left(\sum_{n} e_{\alpha_{n} 1} a_{n}, \sum_{\beta g} e_{\beta g} x_{\beta g}\right)=\sum_{n \beta g} f\left(e_{\alpha_{n} 1}, e_{\beta g}\right) a_{n} x_{\beta g} \\
& =\sum_{\beta}\left(\sum_{\substack{\alpha_{n}<\beta \\
g}} d_{\left(\alpha_{n} \beta g^{-1}\right)} a_{n} x_{\beta g}-\sum_{\substack{\beta<\alpha_{n} \\
g}} d_{\left(\beta \alpha_{n} g\right)} a_{n} x_{\beta g}\right)=0 .
\end{aligned}
$$


There exists $n \in \omega$ such that $\alpha_{n} \notin[x]$. If $\beta g$ is any fixed pair then the last equation $x_{\alpha_{n} y}=0$ for all $y \in H$ force $a_{n} x_{\beta g}=0$, hence $x_{\beta g}=0$ and $x=0$.

If $X$ is a subset of $\widehat{F}$, then $\langle X\rangle$ denotes the $R$-submodule generated by $X$ and $\langle X\rangle_{*}$ is the pure subgroup generated by $X$, more precisely by $\langle X\rangle$, i.e. $\langle X\rangle_{*}=\{y \in \widehat{F}: \exists n \in \mathbb{N}, n y \in\langle X\rangle\}$. Moreover we will use

$$
\langle X\rangle^{*}=\{y \in \widehat{F}: \exists n \in \mathbb{N}, n y \in\langle X, f(X, X)\rangle\}=\langle X, f(X, X)\rangle_{*} .
$$

In many cases $\langle X\rangle_{*}$ and $\langle X\rangle^{*}$ are $R$-submodules as well, which is shown in the next lemma.

Lemma 2.5. If $y \in \widehat{F}$ and $A=A^{*} \subseteq \widehat{F}$, define $B=\langle A, y\rangle^{*}$. Then we have

(a) $B$ is a pure R-submodule of $\widehat{F}$.

(b) $B$ is $f$-invariant: $f(B, B) \subseteq B$.

(c) If $b \in B$, then $n b \equiv y r+f(a, y)+f(y, y t) \bmod A$ for some $a \in A$, $n \in \mathbb{N}$, and $r, t \in R$.

Proof. We begin with (c): If $b \in B$, clearly

$$
\begin{aligned}
n b & \equiv y r+f\left(a+y r, a^{\prime}+y r^{\prime}\right) \\
& \equiv y r+f\left(a r^{\prime *}-a^{\prime} r^{*}, y\right)+f\left(y, y r^{\prime} r^{*}\right) \bmod A
\end{aligned}
$$

for some $a, a^{\prime} \in A, r, r^{\prime} \in R$ and $n \in \omega$, and (c) follows immediately.

(a) From $A=A^{*}$ it follows that $A$ is an $R$-submodule of $\widehat{F}$. If $b \in B$ as above and $s \in R$, then multiplication with $s$ leaves $A$ invariant and

$$
f(v, w) s=f(v, w)\left(s^{\#}\right) \quad\left(\text { in } \widehat{F}_{0}\right) .
$$

Hence $n(b s) \equiv y r s+f(a, y)\left(s^{\#}\right)+f(y, y t)\left(s^{\#}\right) \equiv y r s+f(a s, y)+f(y s, y t)$ $\bmod A$ by (c), and $b s \in B$, which implies (a).

(b) Let $b \in B$ be as in (c) and $b^{\prime} \in B$ with

$$
n^{\prime} b^{\prime}=y r^{\prime}+f\left(a^{\prime}, y\right)+f\left(y, y t^{\prime}\right)+a_{1} \quad\left(a_{1} \in A\right)
$$

from (c). Then we have $a_{2} \in A$ such that

$$
\begin{aligned}
n n^{\prime} f\left(b, b^{\prime}\right) & =f\left(n b, n^{\prime} b^{\prime}\right) \\
& =f\left(y r+f(a, y)+f(y, y t)+a_{2}, y r^{\prime}+f\left(a^{\prime}, y\right)+f\left(y, y t^{\prime}\right)+a_{1}\right) \\
& =f\left(y r, y r^{\prime}\right)+f\left(y r, a_{1}\right)+f\left(a_{2}, y r^{\prime}\right)+f\left(a_{2}, a_{1}\right) \in B .
\end{aligned}
$$

We conclude $f\left(b, b^{\prime}\right) \in B$ and (b) holds.

If $X \subseteq \widehat{F}_{1}$ then we denote $f_{X}=\left\{f_{x}: x \in X\right\}$ where $f_{x}: \widehat{F}_{1} \rightarrow \widehat{F}_{0}$ is the homomorphism $f(-, x)$. Moreover we will identify $f_{x}$ with $f_{x} \uparrow F_{1}$ and all its unique extensions between $F_{1}$ and $\widehat{F}_{1}$ or $F$ and $\widehat{F}$, respectively. Recall that End $X$ denotes the $\mathbb{Z}$-endomorphism ring of $X$. The main result of $\S 4$ will give an abelian group $G$ sandwiched between $F$ and $\widehat{F}$ with

(**) End $G=R \oplus f_{G}$, where $f_{G}$ is a two-sided ideal of End $G$ and $R \cap f_{G}$ $=0$.

Next we will derive consequences of $(* *)$. 


\section{NILPOTENT GROUPS FROM End $G=R \oplus F_{G}$}

(a) The general construction. Suppose $G=G^{+}$satisfies

(**) $F \subseteq G \subseteq \widehat{F}$, such that End $G=R \oplus f_{G}$ and $R=S[H]$,

where $S$ is a commutative ring with only trivial idempotents. The nilpotent group $G^{*}=G_{f}^{*}$ on $G^{+}$is defined by

$$
a . b=a+b+f(a, b) \quad \text { for all } a, b \in G
$$

and $G^{*}$ is nilpotent of class $2(\S 1)$.

If $\alpha \in \operatorname{Aut}\left(G^{*}\right)$, then $f$ is $\alpha$-invariant and $\alpha \in$ Aut $G^{+}$by Proposition 1.1. We can find $r \in R, f_{g} \in f_{G}$ with $\alpha=r+f_{g}$ from $(* *)$. Since $\alpha \in U$ End $G^{+}$ (where $U \ldots$ denotes the units of $\ldots$ ) and $f_{G}$ is a two-sided ideal of End $G^{+}$, also $r \in U R$. We find $r^{-1} \in R$, hence

$$
\alpha=r+f_{g}=r\left(1+f_{g} r^{-1}\right)=r\left(1+g_{g(r \varphi)}\right) .
$$

Moreover, $f_{a}^{2}=0$ for any $a \in G$ and $1+f_{a}$ is an automorphism of $G^{*}$ by arguments from linear algebra. Hence $1+f_{2 G}=\operatorname{Inn} G^{*} \subseteq 1+f_{G} \subseteq U$ End $G^{*}=$ Aut $G^{*}$, where Inn $G^{*}$ denotes all inner automorphisms of $G^{*}$, cf. $\S 1$. From $r\left(1+f_{g(r \varphi)}\right) \in$ Aut $G^{*}$ and $1+f_{g(r \varphi)} \in$ Aut $G^{*}$ we conclude $r \in$ Aut $G^{*}$. We use Proposition 1.1 once more and have $r \in U R \cap$ Aut $G^{\circ}$, and $U R \cap$ Aut $G^{\circ}=$ $\{r \in U R: f$ is $r$-invariant $\}$. In particular

$$
f\left(x\left(r^{*}\right), y\right)=f(x, y)\left(r^{*}\right)=f(x r, y r)=f\left(x r r^{*}, y\right) \quad \text { for all } x, y \in G^{+} .
$$

The last equation for a suitable $y$ and Lemma 2.4 imply

$$
r r^{*}=r^{\#} \text {. }
$$

We have $r^{*}=r^{-1}\left(r^{*}\right)$, and $r$ commutes with $r^{*}$. The same argument with $r^{*}$ gives $r^{* \#}=r^{*} r^{* *}=r^{*} r=r r^{*}=r^{*}$, and $r \in$ Aut $G^{*}$ if and only if $r^{*} \in$ Aut $G^{*}$. Hence $r^{\#}=r r^{*} \in$ Aut $G^{*}$ and $\left(r^{\#}\right)^{2}=\left(r^{\#}\right)\left(r^{\#}\right)=\left(r^{\#}\right)\left(r^{* \#}\right)=\left(r r^{*}\right)^{\#}=\left(r^{\#}\right)^{\#}=r^{\#}$ and $r^{\#}=\left(r^{\#}\right)^{2} \in U S$. The ring $S$ has only trivial idempotents, hence $r^{\#}=1$. We summarize our results.

Proposition 3.1. Condition (**) provides a nilpotent group $G^{*}$ of class 2 with the following properties.

(a) Aut $G^{*} \cap U R+\left\{r \in U R: x^{\#}=1=r r^{*}\right\}=H^{*} \subseteq$ Aut $G^{*}$.

(b) Aut $G^{*}=\left(1+f_{G}\right) \rtimes H^{*}$ is a semidirect product, where $\operatorname{Inn} G=1+f_{2 G} \triangleleft$ $1+f_{G} \triangleleft$ Aut $G^{*}$, and $1+f_{G}=\operatorname{Stab} G$ where Stab $G=\{\sigma \in$ Aut $G: \sigma \mid z G=$ $1, \bar{\sigma}=\mathrm{id}\}$ where $\bar{\sigma}$ is the induced epimorphism on $G / z G^{*}$.

Remark. $G / \approx G$ is abelian and $\sigma$ (as above) induces an epimorphisms $\bar{\sigma}$ on $G / \approx G^{*}$. Next we determine

(b) $H^{*}=$ Aut $G^{*} \cap U R$ and $1+f_{G}$ for particular rings $R=S[H]$.

Proposition 3.2. If $S=\mathbb{Z}$, then $\left(H=H^{*}\right.$ and $)$ Aut $G^{*}=\operatorname{Stab} G^{*} \rtimes H$, where Stab $G / \operatorname{Inn} G$ is an elementary abelian 2-group [of the same rank as $G$ (as will follow by construction in $\S 4)$ ].

Proof. If $r=\sum_{g} g r_{g} \in H^{*}=$ Aut $G^{*} \cap U R$, then $r_{g} \in \mathbb{Z}$ and $r r^{*}=1$ by (3.1)(a). We derive

$$
1=r r^{*}=\left(\sum_{g} g r_{g}\right)\left(\sum_{h} h^{-1} r_{h}\right)=\sum_{g} g\left(\sum_{x y=g} r_{x} r_{y-1}\right),
$$


and for $g=1$ we have $1=\sum_{x y=1} r_{x} r_{y^{-1}}=\sum_{x} r_{x}^{2}$ in $\mathbb{Z}$. This equation holds exactly if $r_{x}= \pm 1$ for one $x \in G$ and $r_{y}=0$ for all $y \neq x$. Moreover $r^{\#}=1$ from $(3.1)(\mathrm{a})$. If $r=-x$, then $1=r^{\#}=(-x)^{\#}-1$ which is impossible, hence $r=x \in G$.

We derive $H^{*} \subseteq H$, and trivially $H^{*}=H$. The rest of (3.2) is automatic by $\S 3(\mathrm{a})$ and $(3.1)(\mathrm{b})$. Recall that $\operatorname{Stab} G^{*}$ is abelian by [9 or 10].

Proposition 3.3. If $S=\mathbb{Z}_{(2)}=\left\{x 2^{n}: x, n \in \mathbb{Z}\right\}$, then Inn $G^{*}=\operatorname{Stab} G^{\cdot}$ and $H^{*}=\left\{r \in U \mathbb{Z}_{(2)}[H]: r r^{*}=1=r^{\#}\right\} \supseteq H$. We have Aut $G^{*}=H^{*} \rtimes \operatorname{Inn} G$.

Proof. The abelian group $G^{+}$is 2 -divisible because $2 S=S$, hence $1+f_{2} G=$ $1+f_{G}$ and the rest follows from (3.1) and (1.3).

Starting with suitable groups $H$, with particular finite $H$ for instance, then it is possible to solve the equations $r r^{*}=1=r^{\#}$ which determine $H^{*}$. Hence (3.3) is a good tool to calculate outer automorphism groups $H^{*}=$ Aut $G^{*} /$ Inn $G^{*}$ of nilpotent groups of class 2 . There are clearly many finite groups among the $H^{*}$ 's not belonging to Corner's [2] classification of all finite groups realizable as (outer) automorphism groups of torsion-free abelian groups; see $\S 5$ for more details.

\section{COMBINATORIAL METHODS FOR CONSTRUCTING $G$ WITH End $G=R \oplus f_{G}$}

(a) Combinatorial tools. The combinatorial methods derive from model theory, and the basic is due to S. Shelah [21] and named (Shelah's) Black Box in [3]. These ideas have been exploited in abelian group theory as well as in ring and field theory, see $[3,6,8]$ and references in [3]. Here we adjust the algebraic tools and refer to [3] for a proof of the adjusted Black Box.

Let $\kappa<\lambda$ be infinite cardinals with $\lambda^{\kappa}=\lambda,|R|=|S| \cdot|H| \leq \lambda$, and $|H| \leq \kappa$. Then we define $T$ and $F$ as in $\S 2$ and let $f: \widehat{F} \times \widehat{F} \rightarrow \widehat{F}$ be the alternating $S$-bilinear map given in (2.2). Recall the definition of $f_{g}=f(-, g): \widehat{F} \rightarrow \widehat{F}$ for any $g \in \widehat{F}$, and essentially we have $f_{g}: \widehat{F}_{1} \rightarrow \widehat{F}_{0}$. We want to construct

$$
F \subseteq G \subseteq \widehat{F} \text { such that End } G=R \oplus f_{G} .
$$

First we adjust the notations and results from [3, $\S 3$ and Appendix].

Recall that the cofinality of $\lambda$ is the smallest cardinal $\operatorname{cf}(\lambda)$ such that a continuous, strictly increasing function $\rho: \operatorname{cf}(\lambda)+1 \rightarrow \lambda+1$ with $\rho(0)=0$ and $\rho(\operatorname{cf}(\lambda))=\lambda$ exists. Let $\rho$ be a fixed function with these properties. Next we define a norm of a knot $\tau$ of the tree $T$ to be

$$
\|\tau\|=\min \left\{\nu \in \operatorname{cf} \lambda: \tau \in{ }^{\omega>} \rho(\nu)\right\} .
$$

Hence $\|\tau\|$ is an ordinal $<\operatorname{cf}(\lambda)$, in fact $\|\tau\|$ is the smallest ordinal such that the ordinal sequence $\tau$ can be constructed by ordinals $<\rho(\operatorname{cf}(\lambda))$. Using the support $(\S 2)$, the norm can easily be extended to subsets of $\widehat{F} ; \mathrm{cf}$. [3, p. 451]. If $x \in \widehat{F}$, then $\|x\|=\sup \{\|\tau\|: \tau \in[x]\}$.

In order to work with not too may homomorphisms, we restrict their domains drastically to the size $\leq \kappa$ and define a trap as a triple $(e, P, \varphi)$ where

(i) $e:{ }^{\omega>} \omega \rightarrow T={ }^{\omega>} \lambda$ is a tree embedding.

(ii) $P$ is a canonical summand of $F$. This means that there is a subset $X \subset T$ with $|X| \leq \kappa$ and 


$$
P=\left\langle e_{\tau g}, d_{\alpha \beta h}: \tau g \in X \times H, \alpha \beta h \in X \times X \times H \text { with } \alpha \leq \beta\right\rangle^{*},
$$

see $\S 2$.

(iii) $X$ in (ii) is a subtree of $T$ with $\mathrm{cf}\|P\|=\omega$.

(iv) $\|v\|=\|P\|$ for all branches $v$ in $\operatorname{Im} e$. A branch $v$ in $T$ is a maximal linear subset of $T$, which we may identify with a map $v: \omega \rightarrow \lambda$ and $v=\{v$ | $n: n \in \omega\}$.

(v) $\varphi \in$ End $P$.

If $X \subseteq T$, then $\operatorname{Br} X$ will denote all branches of $T$ in $X$, moreover $\operatorname{Br} Y=\operatorname{Br}[Y]$ for any subset $Y \subseteq \widehat{F}$. Because of $(* *)$, we have to deal with unwanted endomorphisms of $\widehat{F}$, and the idea is to do this looking at one partial endomorphisms at a time. In order to make sure that all endomorphisms have been inspected, the restriction of sufficiently many of them is enumerated in a special way by Shelah's

(4.1) Black Box. Suppose $f, \widehat{F}$, and $R=S[H]$ are given as above $|R| \leq \lambda$, $|H| \leq \kappa$, and $\lambda^{\kappa}=\lambda$. For some ordinal $\lambda^{*}$ of cardinality $\lambda$ there exists $a$ transfinite sequence of traps $\left(e_{\alpha}, P_{\alpha}, \varphi_{\alpha}\right)\left(\alpha \in \lambda^{*}\right)$ such that, for $\alpha, \beta \in \lambda^{*}$,

(a) $\beta<\alpha \Rightarrow\left\|P_{\beta}\right\| \leq\left\|P_{\alpha}\right\|$.

(b) $\beta \neq \alpha \Rightarrow \operatorname{Br}\left(\operatorname{Im} e_{\alpha}\right) \cap \operatorname{Br}\left(\operatorname{Im} e_{\beta}\right)=\varnothing$.

(c) $\beta+2^{\aleph_{0}} \leq \alpha \Rightarrow \operatorname{Br}\left(\operatorname{Im} e_{\alpha}\right) \cap \operatorname{Br}\left(P_{\alpha}\right)=\varnothing$.

(d) If $X \subseteq \widehat{F}$ has cardinality $|X| \leq \kappa$, and $\varphi \in$ End $\widehat{F}$, there exists $\alpha<\lambda^{*}$ such that $X \subseteq \widehat{P}_{\alpha},\|X\|<\left\|P_{\alpha}\right\|, \varphi \mid \widehat{P}_{\alpha}=\varphi_{\alpha}$.

Proof. See [3, pp. 475-478], which is a counting argument only.

(b) Construction of the group $G$. Choose a transfinite sequence $\left(e_{\alpha}, P_{\alpha}, \varphi_{\alpha}\right)$ $\left(\alpha \in \lambda^{*}\right)$ as in (4.1). As $\lambda^{*}$ is an ordinal, we can construct $G$ as the union of an ascending continuous chain of submodules $G_{\alpha}(\alpha<\mu)$ of $\widehat{F}$ and $\mu<\lambda^{*}$ using transfinite induction on $\mu$. At the same time we will decompose the ordinals $<\lambda^{*}$ inductively into two disjoint subsets $\lambda_{w}^{*}$ and $\lambda_{s}^{*}$ depending on the quality of the homomorphism $\varphi_{\alpha}$ related with $\alpha$ by the Black Box. At the end we will show that there are no endomorphisms of $G$ extending partial endomorphisms $\varphi_{\alpha}$ with $\alpha \in \lambda_{s}^{*}$ and this will lead immediately to the desired condition $(* *)$. At stage $\mu$ we will use information of the $G_{\alpha}(\alpha<\mu)$ which are already constructed and of the traps $\left(e_{\alpha}, P_{\alpha}, \varphi_{a}\right) \quad(\alpha \leq \mu)$ given by the Black Box.

We begin the induction with $G_{0}=F$ and let $\mu \leq \lambda^{*}$. Assume that we have found ascending chains of submodules $G_{\beta}$ of $\widehat{F}$ and two chains of subsets $\beta_{w}, \beta_{s}$ of $\lambda^{*}$ with $\beta=\beta_{w} \cup \beta_{s}$ for all $\beta<\mu$ and elements $g_{\beta} \in \widehat{F}(\beta+1<\mu)$ such that the following holds:

$\left(\alpha_{1}\right) g_{\beta} \varphi_{\beta} \notin G_{\alpha}$ for all $\beta \in \mu_{s}, \alpha<\mu$.

$\left(\alpha_{2}\right) \quad g_{\beta}=x_{\beta}+v_{\beta}$ with $\left\|v_{\beta}\right\|>\left\|x_{\beta}\right\|$ and $v_{\beta} \in \widehat{F}_{1}$ is a branch element, i.e. $\exists v_{\beta}^{\prime} \in \operatorname{Br}\left(\operatorname{Im} e_{\beta}\right)$ and $v_{\beta}=\sum_{n \in \omega} e_{v_{\beta}^{\prime}(n)} . q_{n}$ for a sequence $q_{n} \in \mathbb{Z}=\langle 1\rangle \subseteq S$ converging to 0 in the $\mathbb{Z}$-adic topology (compare [3, p. 453]).

$\left(\beta_{3}\right) \quad \beta \in \mu_{w}$ if and only if for all choices $g=g_{\beta} \in \widehat{F}$ with $\left(\alpha_{1}\right),\left(\alpha_{2}\right)$ it would follow that $g \varphi_{\beta} \in\left\langle G_{\beta}, g\right\rangle^{*}$.

In this case we say that $\beta$ is a weak ordinal, and $\beta$ is strong otherwise. If $\mu$ is a limit ordinal, we simply take $G_{\mu}$ and $\mu_{s}, \mu_{w}$ to be the union of all 
$G_{\beta}, \beta_{s}$ and $\beta_{w}(\beta<\mu)$ respectively. [Elements $g_{\mu}$ are not defined for limit ordinals $\mu$, and the inductive requirements are automatically fulfilled.]

Now we come to the case $\mu=\beta+1$ of a successor ordinal $\mu$. If we can find $g_{\beta}$ satisfying all the inductive requirements for a strong ordinal $\beta$, then we take this element and let $G_{\mu}=\left\langle G_{\beta}, g_{\beta}\right\rangle^{*}$. Moreover we put $\beta$ into $\mu_{s}$ and observe that $g_{\beta} \notin G_{\mu}=G_{\beta+1}$ in this case.

If such an element $g_{\beta}$ does not exist, we put $\beta$ into $\mu_{w}$ and let $G_{\mu}=$ $\left\langle G_{\beta}, g_{\beta}\right\rangle^{*}$, where $g_{\beta}$ is now an element which satisfies only the (weaker) requirements $\left(\alpha_{1}\right)$ and $\left(\alpha_{2}\right)$. In this case we call $\beta$ a weak ordinal. It is not obvious, that one of the two choices is always possible; condition $\left(\alpha_{1}\right)$ must be checked!

However, the proof given in [3, p. 458, proof of Corollary 3.10 , which is based on (3.9)] shows that this is the case indeed; a third class of ("useless") ordinals is not needed. The weak ordinals $\beta$ are necessary in order to continue the construction for endomorphisms $\varphi_{\beta}$ which extend to endomorphisms in $R \oplus f_{G}$ like $\varphi_{\alpha}=\mathrm{id}$. We conclude from this

$$
\alpha \in \lambda_{s}^{*} \quad \text { if and only if } \quad g_{\alpha} \varphi_{\alpha} \notin G,
$$

where $G=\bigcup_{\alpha<\lambda^{*}} G_{\alpha}$ and $\lambda^{*}=\lambda_{w}^{*} \dot{\cup} \lambda_{s}^{*}$.

Next we will show the main result of this section.

(c) End $G=R \oplus f_{G}$.

Each $G_{\alpha}$ is a pure $R$-submodule of $\widehat{F}$ by $(2.5)(\mathrm{a})$, moreover $f_{G_{\alpha}}\left(G_{\alpha}, G_{\alpha}\right) \subseteq$ $G_{\alpha}$ by $(2.5)(\mathrm{b})$. We conclude

(a) $R+f_{G} \subseteq$ End $G$ and,

(b) $G$ is a cotorsion-free abelian group which follows similar to [3, Lemma 6.2, p. 464].

It remains to show that

(c) End $G \subseteq R+f_{G}$.

(d) First we consider $\varphi \in$ End $G$ such that $n \varphi \notin R+f_{G}$ for all $n \in \mathbb{N}$.

By (b) and the argument in [3, Lemma 4.5, p. 459] we can find a canonical summand $P \subseteq F$ such that

(e) for any $n \in \mathbb{N}, r \in R$, and $g \in G$ there is $x=e_{\gamma h} \pi \in \widehat{P} \quad(\pi \in \widehat{\mathbb{Z}})$ with $x\left(n \varphi-r+f_{g}\right) \notin G$.

[The assertion (e) says that the "bad action" of $\varphi$ which is $n \varphi \notin R+f_{G}$ is recognizable on "small" canonical summands.]

Using the Black Box, we can find $\alpha<\lambda^{*}$ such that $P \subset \widehat{P}_{\alpha},\|P\|<\left\|P_{\alpha}\right\|$, and $\varphi \uparrow \widehat{P}_{\alpha}=\varphi_{\alpha}$. We want to show that $\alpha \in \lambda_{s}^{*}$, hence $g_{\alpha} \varphi=g_{\alpha} \varphi_{\alpha} \notin G$. From $g_{\alpha} \in G$ then follows $\varphi \notin$ End $G$, which is a contradiction.

Suppose $\alpha$ is not strong. We want to derive a contradiction from (e). Then (c) will be shown. The idea is to find candidates " $g_{\alpha}$ " which could have been used at stage $\alpha$ of the construction to show that $\alpha$ is a strong ordinal indeed. Then we know from the construction that (possibly a different element) $g_{\alpha}$ with $g_{\alpha} \varphi_{\alpha} \notin G$ has been used at stage $\alpha$.

First we pick a branch element $v_{\alpha}$ in $\operatorname{Im}\left(e_{\alpha}\right)$ as a $g_{\alpha}$. The ordinal $\alpha$ is assumed to be weak, hence

$$
v_{\alpha} \varphi_{\alpha} \in\left\langle G_{\alpha}, v_{\alpha}\right\rangle^{*}=G_{\alpha+1}
$$

and, by $(2.5)(\mathrm{c})$, 


$$
v_{\alpha} n \varphi_{\alpha} \equiv v_{\alpha} r+f\left(g, v_{\alpha}\right)+f\left(v_{\alpha}, v_{\alpha} t\right) \bmod G_{\alpha}
$$

for some $g \in G_{\alpha}, n \in \mathbb{N}, r, t \in R$.

Next we choose $x \in \widehat{P}_{\alpha}$ as in (e)

$$
x=e_{\gamma h} \pi \quad \text { and } \quad x\left(n \varphi_{\alpha}-r+f_{g}\right) \notin G
$$

and try another element to test weakeness of $\alpha$ :

$$
g_{\alpha}=x+v_{\alpha} .
$$

Observe that $\left(\alpha_{2}\right)$ is automatically fulfilled, and $\left(\beta_{3}\right)$ follows from the construction as mentioned in $(*)$. Hence we can concentrate on $\left(\alpha_{1}\right)$. As $\alpha$ is weak, we conclude $g_{\alpha} \varphi_{\alpha} \in G_{\alpha+1}$, and find another quadruple, $\bar{g}, \bar{n}, \bar{r}, \bar{t}$ such that

(h) $\left(x+v_{\alpha}\right) \bar{n} \varphi_{\alpha} \equiv\left(x+v_{\alpha}\right)+f\left(\bar{g}, x+v_{\alpha}\right)+f\left(x+v_{\alpha},\left(x+v_{\alpha}\right) \bar{t}\right) \bmod G_{\alpha}$.

Multiplying (f) and (h) with $\bar{n}$ and $n$ respectively, subtraction and (2.3) $f(x, x \bar{t})=0$ gives

$$
\begin{aligned}
x n \bar{n} \varphi_{\alpha} \equiv & x \bar{r} n+v_{\alpha}(\bar{r} n-r \bar{n})+f(\bar{g} n, x)+f\left(\bar{g} n-g \bar{n}, v_{\alpha}\right)+f(x, x \bar{t}) n \\
& +f\left(v_{\alpha}, x \bar{t} n-v_{\alpha} t \bar{n}-x \bar{t}^{*} n+v_{\alpha} \bar{t} n\right) \\
\equiv & x \bar{r} n+v_{\alpha}(\bar{r} n-r \bar{n})+f(\bar{g} n, x)+f(x, x \bar{t}) \\
& \left.\quad+f\left(v_{\alpha},(g \bar{n}-\bar{g} n)\right)+x\left(\bar{t} n-\bar{t}^{*} n\right)+v_{\alpha}(\bar{t} n-t \bar{n})\right) \\
\equiv & x \bar{r} n+v_{\alpha}(\bar{r} n-r \bar{n})+f(\bar{g} n, x) \\
& +f\left(v_{\alpha},(g \bar{n}-\bar{g} n)+x\left(\bar{t} n-\bar{t}^{*} n\right)+v_{\alpha}(\bar{t} n-t \bar{n})\right) \quad \bmod G_{\alpha} .
\end{aligned}
$$

The norm of $v_{\alpha}$ is strictly larger than the norm of $x n \bar{n} \varphi_{\alpha}$, and if $(\bar{r} n-r \bar{n}) \neq$ 0 then $v_{\alpha}(\bar{r} n-r \bar{n})$ has support almost disjoint of the support of the other elements of the last equation. We conclude $\bar{r} n=r \bar{n}$ and modulo $G_{\alpha}$

$$
\left.x n \bar{n} \varphi_{\alpha} \equiv x r \bar{n}+f(\bar{g} n, x)+f\left(v_{\alpha},(g \bar{n}-\bar{g} n)\right)+x\left(\bar{t} n-\bar{t}^{*} n\right)+v_{\alpha}(\bar{t} n-t \bar{n})\right) .
$$

The definition of $f$ and a similar support argument force that $f\left(v_{\alpha}, v_{\alpha}(\bar{t} n-t \bar{n})\right)$ must be 0 , and the equation reduces to

$$
x n \bar{n} \varphi_{\alpha} \equiv x r \bar{n}+f(\bar{g} n, x)+f\left(v_{\alpha}, g \bar{n}-\bar{g} n+x\left(\bar{t} n-\bar{t}^{*} n\right)\right) \quad \bmod G_{\alpha} .
$$

A final support argument implies

$$
f\left(v_{\alpha}, g \bar{n}-\bar{g} n+x\left(\bar{t} n, \bar{t}^{*} n\right)\right)=0 .
$$

The branch element $v_{\alpha}$ satisfies the hypothesis of Lemma 2.4 , hence

$$
g \bar{n}-\bar{g} n+x\left(\bar{t} n-\bar{t}^{*} n\right)=0
$$

from (2.4). Solving this for $\bar{g} n$ and substituting in the congruence for $x n \bar{n} \varphi_{\alpha}$, we derive from $(2.3)$ that

$$
x n \bar{n} \varphi_{\alpha} \equiv x r \bar{n}+f(g \bar{n}, x)+f\left(x\left(\bar{t} n-\bar{t}^{*} n\right), x\right) \equiv x r \bar{n}-x f_{g} \bar{n} \quad \bmod G_{\alpha} .
$$

The group $G$ is torsion-free and $G_{\alpha}$ is pure in $G$, hence

$$
x n \varphi_{\alpha} \equiv x r-x f_{g} \quad \bmod G_{\alpha}
$$


and $x\left(n \varphi_{\alpha}-r+f_{g}\right) \in G_{\alpha}$ contradicts $(\mathrm{g})$. Hence $\alpha$ must be strong and as we said at the beginning of this proof, this implies $\varphi \notin$ End $G$, contradicting (d).

Otherwise, there exists an $n \in \mathbb{N}$ such that $n \varphi \in R+f_{G}$. Hence $n \varphi=r+f_{g}$ for some $r \in R, g \in G$. Consider any "basic" element $x=e_{\beta 1}$ for some $\beta \in T$. We have $x n \varphi=x r+f(x, g)$.

Projecting both sides of the last equation to $F_{1}$, it follows trivially that $r=$ $n . s$ for some $s \in R$. We derive $f_{g}=n(\varphi-s)$ and $n$ divides $f(x, g)$, which forces $g=n g^{\prime}$ for some $g^{\prime} \in G$. We conclude $\varphi=s+f_{g^{\prime}} \in R+f_{G}$ and End $G=R+f_{G}$ follows.

If $\sigma \in R \cap f_{G}$, then apply $\sigma$ to the same $x=e_{\beta 1}$. Hence $x \sigma=x r=f(x, g)$ which must be 0 by definition of $f$, and (**) is shown.

\section{REALIZING $Z_{3}$ AS OUTER AUTOMORPHISM GROUP}

If $G$ is torsion-free abelian, then any torsion group $A=$ Aut $G=$ Out $G$ belongs to a complete list of very special groups, which is due to Corner [2], cf. $[4$, p. 335]:

(a) $A$ is a subdirect product of primordial groups.

(b) If $Z_{2}$ is not a summand of $A$, then there exists $a \in A$ with centralizer $\mathfrak{r}_{A}(a)$ is a 2-group and $\left\langle x \in A: x^{3}=1\right\rangle$ is a direct product of groups whose 3-Sylow-group is either 1 or $Z_{3}$.

Obviously $Z_{3}$ is not an (outer) automorphism group of any torsion-free abelian group. Hence it is interesting to see that $Z_{3} \cong$ Out $G$ for (a class of) torsion-free nilpotent groups of class 2. This also proves that (3.3) is useful to determine Out $G$ as indicated at the end of $\S 3$. We apply (3.3) and determine $H^{*}=\{r \in$ $\left.U \mathbb{Z}_{(2)}\left[Z_{3}\right]: r r^{*}=1=r^{\#}\right\}$.

Consider any $r \in H^{*} \backslash Z_{3}$, hence $r=\alpha .0+\beta .1+\gamma .2$ in $\mathbb{Z}_{(2)}\left[Z_{3}\right]$ with $\alpha, \beta, \gamma \in \mathbb{Z}_{(2)}$, and $r r^{*}=1=r^{\#}$. We derive

(1) $\alpha^{2}+\beta^{2}+\gamma^{2}=1$,

(2) $\alpha \beta+\beta \gamma+\alpha \gamma=0$, from $r r^{*}=1$ and

(3) $\alpha+\beta+\gamma=1$, from $r^{\#}=1$.

Using properties (2) and (3) we have $\beta^{2}+\beta(\alpha-1)+\alpha^{2}-\alpha=0$ which implies $(\beta+(\alpha-1) / 2)^{2}=\frac{1}{4}\left(2 \alpha-3 \alpha^{2}+1\right)$ and $2 \alpha-3 \alpha^{2}+1$ is a square in $\mathbb{Z}_{(2)}$. Hence $-\frac{1}{3}\left[(3 \alpha-1)^{2}-4\right]$ is a square as well. Now suppose $\alpha \neq 0$ and let $\alpha=s .2^{-n}$, $s$ odd and observe that $n>0$ from $r \notin Z_{3}$. We derive that

$$
-\left(\frac{1}{3}\left(3 s-2^{n}\right)^{2}-2^{2(n+1)}\right) \text { is a square in } \mathbb{Z}_{(2)}
$$

and thus a square in $\mathbb{Z}$. An easy calculation shows that $\left(2^{n}-s\right)\left(2^{n}+3 s\right)$ must be a square in $\mathbb{Z}$. Since $s$ is odd, the $\operatorname{gcd}\left(2^{n}-s, s\right)=\left(2^{n}, s\right)=1$ and $2^{n}-s=a^{2}$ and $2^{n}+3 s=b^{2}$ are squares in $\mathbb{Z}$. Now $-3 a^{2} \equiv b^{2} \bmod 2^{n+2}$ and $a, b$ are units $\bmod 2^{n+2}$. In particular -3 must be a square residue $\bmod 2^{n+2}$. However, $n+2 \geq 3$ implies $-3 \equiv 1 \bmod 8$ a contradiction; cf. LeVeque [15, p. 64]. We derive $(3 \alpha-1)^{2}-4=0$ and $\alpha \in\left\{1,-\frac{1}{3}\right\}$. Now $\alpha=\frac{1}{3} \notin \mathbb{Z}_{(2)}$ is impossible, hence $\alpha=1$ and then $\gamma=\beta=0$ and $r \in Z_{3}$.

If $\alpha=0$, then from (2) and (3), $\{\beta, \gamma\}=\{0,1\}$, which is absurd since $r \notin \mathbb{Z}_{(3)}$. 


\section{REFERENCES}

1. R. Baer, Groups with Abelian central quotient groups, Trans. Amer. Math. Soc. 44 (1938), 357-386.

2. A. L. S. Corner, Groups of units of orders in Q-algebras, Abelian Group Theory, Proc. Caribbean Conf. 1991 (L. Fuchs and R. Göbel, eds.), (to appear).

3. A. L. S. Corner and R. Göbel, Prescribing endomorphism algebras, a unified treatment, Proc. London Math. Soc. (3) 50 (1985), 447-479.

4. M. Dugas and R. Göbel, Every cotorsion-free ring is an endomorphism ring, Proc. London Math. Soc. (3) 45 (1982), 319-336.

5. and results on ULF groups, submitted 1990.

6. __ Torsion-free nilpotent groups and E-modules, Arch. Math. 54 (1990), 340-351.

7. __, Outer automorphism groups, Illinois J. Math. 35 (1991), 27-46.

8. __, All finite groups are Galois groups over any field, Trans. Amer. Math. Soc. 304 (1987), 355-384.

9. P. Hall, Some sufficient conditions for a group to be nilpotent, Illinois J. Math. 2 (1958), 787-801.

10. P. Hali and B. Hartley, The stability group of a series of subgroups, Proc. London Math. Soc. (3) 16 (1966), 1-39.

11. H. Heineken, Automorphisms groups of torsion-free nilpotent groups of class two, Symposia Math. 17 (1976), 235-250.

12. H. Heineken and H. Liebeck, The occurrence of finite groups in the automorphism group of nilpotent groups of class 2, Arch. Math. 25 (1974), 8-16.

13. S. Kojima, Isometry transformation of hyperbolic 3-manifolds, Topology Appl. 29 (1988), 297-307.

14. M. Lazard, Sur les groupes nilpotents et les anneaux de Lie, Ann. Sci. École Norm. Sup. (Pisa) (3) 71 (1954), 101-190.

15. W. J. LeVeque, Topics in number theory, Vol. I, Addison-Wesley, Reading, Mass., 1961.

16. S. Mac Lane, Homology, Springer, Berlin, 1963.

17. T. Matumoto, Any group can be represented by an outer automorphism group, Hiroshima Math. J. 19 (1989), 209-219.

18. M. R. Pettet, On inner automorphisms of finite groups, Proc. Amer. Math. Soc. 105 (1989), $1-4$.

19. __ Characterizing inner automorphisms of groups, manuscript.

20. P. E. Schupp, A characterization of inner automorphisms, Proc. Amer. Math. Soc. 101 (1987), 226-228.

21. S. Shelah, A combinatorial principle and endomorphism rings. I: On p-groups, Israel J. Math. 49 (1984), 239-257.

22. R. B. Warfield, Jr., Nilpotent groups, Lecture Notes in Math., vol. 513, Springer, 1976.

23. U. H. M. Webb, The occurrence of groups as automorphism groups of nilpotent p-groups, Arch. Math. 37 (1981), 481-498.

24. A. E. Zalesskii, An example of a torsion-free nilpotent group having no outer automorphisms, Mat. Zametki 11 (1972), 21-26; Math. Notes 11 (1972), 16-19.

Department of Mathematics, Baylor University, Waco, Texas 76793

E-mail address: dugasm@baylor

FB6, Mathematik, Universität Essen GHS, D 4300 Essen 1, Germany

E-mail address: mat100@deohrzla 